\title{
Prognostic Value of Systemic Immune Index in Patients with Metastatic Gastric Cancer
}

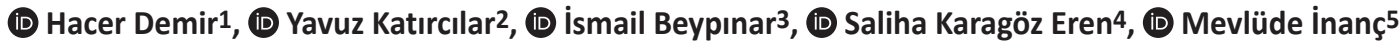 \\ ${ }_{1}^{1}$ Afyon Health Sciences University Faculty of Medicine, Department of Internal Medicine and Medical Oncology, Afyonkarahisar, Turkey \\ 2Kayseri City Training and Research Hospital, Clinic of Internal Medicine, Kayseri, Turkey \\ 3 University of Health Sciences Turkey, Eskişehir City Hospital, Clinic of Internal Medicine and Medical Oncology, Eskişehir, Turkey \\ ${ }^{4}$ Kayseri City Training and Research Hospital, Clinic of General Surgery, Kayseri, Turkey \\ ${ }^{5}$ Erciyes University Faculty of Medicine, Department of Internal Medicine and Medical Oncology, Kayseri, Turkey
}

\section{Abstract}

Objective: Immune indexes are used to predict prognosis and survival in patients with cancer. Systemic immune-inflammation index (SII) is an index that is calculated using neutrophils $(\mathrm{N})$, lymphocytes $(\mathrm{L})$, and platelet $(\mathrm{P})$ counts (SII=N x P/L). SII is shown to be associated with prognosis in many tumors. The objective of this study is to evaluate the relationship between SII and prognosis in patients with metastatic gastric cancer.

Methods: In this study, the data of 187 patients who were followed up with the diagnosis of metastatic gastric cancer in the medical oncology outpatient clinic of the tertiary training and research hospital were retrospectively reviewed. SII was calculated using the formula $\mathrm{N} x \mathrm{P} / \mathrm{L}$ and the optimal cut-off was determined as 600 , which was the median value. Values below 600 were grouped as low SII and values above 600 were grouped as high SII. The effect of SII on survival was evaluated by the log-rank test and the Kaplan-Meier curve.

Results: For the study, medical files of 253 patients who were diagnosed with metastatic gastric cancer were scanned. Patients who had no follow-up and whose pre-treatment hemogram data were not available at that time were excluded. Finally, this study included 187 patients. In total, there were 63 (33.7\%) female and 124 (66.3\%) male patients, with a median age of 63 (27-88) years. Although survival was shorter in patients with SII above 600, no statistical significance was detected ( 9 months vs. 12 months; $p=0.13$ ). In primary surgically resected patients, survival was significantly better (13 months vs. 4 months $p<0.001)$, but there was no difference in SII between the operated and non-operated groups.

Conclusion: Our study suggests that a high SII may be a poor prognostic factor in patients with metastatic gastric cancer, despite no statistical significance. Well-designed prospective studies with a larger number of patients are needed to evaluate the prognostic significance of SII and determine the optimal cut-off value before its inclusion into the clinical routine in this group of patients.

Keywords: Gastriccancer, prognosis, systemicimmune-inflammation index (SII)

\section{INTRODUCTION}

Gastric cancer is the fifth most common cancer in the world and ranks third among all cancer-related deaths. Globally, more than one million patients were diagnosed with gastric cancer in 2018, resulting in over 780,000 deaths. Despite the decreasing incidence and mortality of gastric cancer in recent years, it is still a major public health challenge $(1,2)$. The highest cancer incidence in the world is found in East Asia, Eastern Europe, and South America, followed by Japan and Korea, where the incidence is also quite high (3).

Gastric cancer is still a high mortality disease with a poor prognosis despite the new developments in treatment such 
as new surgical techniques, chemotherapy, radiotherapy, and immunotherapy (4). Because gastric tumors exhibit late clinical manifestations, they are usually diagnosed at an advanced stage, with only around $10 \%$ of patients diagnosed at an early stage with the five-year survival rate of $10-30 \%(5,6)$.

Surgery combined with adjuvant chemotherapy and radiotherapy when diagnosed at an early stage increases survival chances, whereas systemic chemotherapy and radiotherapy aim to improve the survival and palliation of symptoms in patients diagnosed at the metastatic stage $(5,7)$.

Systemic inflammation is known to play an important role in the development of many cancers, including gastric cancer. Chronic inflammation and chronic atrophic gastritis, especially due to Helicobacter pylori infection, increase the developmental risk of gastritis cancer $(8,9)$. Cancer-related inflammation is a vital component of the tumor microenvironment, and inflammatory cells play an essential role in tumor development and progression. Researchers have shown that systemic inflammatory responses include DNA damage, angiogenesis, and tumor invasion and migration (10). Furthermore, another group of researchers have assessed the use of some histological and immunological markers in evaluating the prognosis of gastric cancer; however, many of these markers require tissue, cost, and time $(6,11)$. Inflammatory cells such as white blood cells, neutrophils (N), lymphocytes $(\mathrm{L})$, monocytes, platelets $(\mathrm{P})$ and parameters such as $\mathrm{N} / \mathrm{L}$ ratio, monocyte/L ratio, $\mathrm{P} / \mathrm{L}$ ratio, are used routinely and believed to be indicators of systemic inflammatory and recommended as a prognostic factor in many types of cancers $(6,10,12-14)$.

Recently, systemic immune-inflammation index (SII) based on N, $P$ counts, and $L$ counts in the peripheral blood has been used for a better reflection of the balance between host immune response and inflammation. Prognostic value of the results obtained from this calculation has been confirmed in many solid tumors such as colon, esophagus, hepatocellular and lung cancers (15-18).

SII has been reported to be associated with prognosis in patients operated for gastric cancer, as well as in patients with locally advanced gastric cancer who receive neoadjuvant chemotherapy. However, its prognostic use in patients with metastatic gastric cancer is not clear $(6,19)$. The objective of this study is to evaluate whether the systemic immune index has a prognostic significance in patients with metastatic gastric cancer.

\section{METHODS}

Our study included 187 patients who were diagnosed with pathologically confirmed metastatic gastric cancer between 2006 and 2017 at the Medical Oncology Clinic of Kayseri Training and Research Hospital. In addition, their pre-treatment hemogram values and follow-up data were also recorded. Patients with steroid use that may affect hemogram parameters or patients with autoimmune disease, systemic inflammatory disease, and active infection at the time of diagnosis were not included in the study. Consent was obtained from the patients that the file information would be used before the study. Participating patients' gender, age, pathological tumor type, metastasis regions, type of surgery, hemogram, and tumor marker levels were then determined and evaluated. SII was calculated with the hematological parameters of the patients with the formula $\mathrm{P} \times(\mathrm{N} / \mathrm{L})$, and the median SII value of 600 was accepted as the cut-off value. Those with the SII cut-off value $\geq 600$ were considered as high and those with $<600$ as low.

\section{Statistical Analysis}

All analyses were performed using Statistical Package for Social Sciences version 22.0 software (SPSS Inc, Chicago, IL).

Categorical variables in the clinicopathological database were presented as frequency and percentage (\%) and compared using the chi-square test or Fisher exact test. The basic characteristics of the patients were expressed as median for qualitative variables. Independent t-test was used in the analysis of numeric parameters that do not comply with normal distribution and cases who did not have a normal distribution were evaluated using Mann-Whitney U test.

The Kaplan-Meier curve was used for determining the effect of SII on overall survival (OS), whereas the log-rank test was used for the comparison of survival rates.

\section{Ethical Approval}

Ethical approval, dated 23/07/2020/07 decision number 126, was obtained from the Ethics Committee of Kayseri Training and Research Hospital.

\section{RESULTS}

For the study, the medical files of 253 patients who were diagnosed with metastatic gastric cancer were scanned. Patients who had no follow-up and whose pre-treatment hemogram data were not available were excluded. Finally, this study included 187 patients. In total, there were 63 (33.7\%) female and 124 (66.3\%) male patients, with a median age of 63 (27-88) years. Surgery was performed for primary gastric cancer in 150 patients (69.5\%) before staging the patients and the most common surgery was total gastrectomy (37\%). As for the pathological subtypes, adenocarcinoma was the 
most frequently observed at a rate of $73.8 \%$, among which $28.3 \%$ were poorly differentiated, $24.1 \%$ were moderately differentiated, 3.7\% were well-differentiated tumors according to the differentiation status.

Evaluation of the metastasis sites revealed that the most common metastasis sites were liver (25\%) and peritoneum (24\%), whereas $20.3 \%$ of the patients had multiple metastases at the time of diagnosis. Tumor marker elevation was observed in $114(61 \%)$ patients at the time of diagnosis (Table 1).

The median white blood cells, hemoglobin, N, P, and L counts in blood parameters were $7 \times 10^{3} \mu / \mathrm{dL}, 11.9 \mathrm{~g} / \mathrm{dL}, 4.44 \times 10^{3} \mathrm{u} /$ $\mathrm{dL}$, and $1.615 \times 10^{3} \mathrm{u} / \mathrm{dL}$, respectively. A significant relationship was observed between hemogram parameters and SII when the optimum SII cut-off value was determined as the median SII value (Table 2).

Patients were divided into two groups according to SII levels: Those with SII $<600$ were accepted as the low group, and those with SII >600 as the high group. There were 60 patients in the low SII group and 127 patients in the high SII group. Although the median OS was 10 (8.2-11.7) months in the whole study group, there was no significant relationship among age $(p=0.61)$, gender $(p=0.97)$, pathological subtype $(p=0.28)$, serum tumor elevation $(p=0.06)$, and survival. However, survival was statistically significantly better in patients undergoing primary surgery $(\boldsymbol{p}<0.001)$ (Table 3, Figure 1$)$.

Evaluation of the relationship between SII and survival showed that the median OS was 12 (9.5-14.4) months in the low SII

\begin{tabular}{|c|c|c|c|c|c|c|}
\hline Age & $\begin{array}{l}\text { (Mean } \pm \text { SD) } \\
61.6 \pm 12.26\end{array}$ & $\begin{array}{l}\text { Median (min-max) } \\
63(37-75)\end{array}$ & & & & \\
\hline Gender & Male & Female & & & & \\
\hline n (\%) & $124(66.3)$ & $63(33.7)$ & - & - & - & - \\
\hline Histologic subtype & Signet ring cell & Adenocarcinoma & Mucinous & Mixed & & \\
\hline n (\%) & $46(24.6)$ & $138(75.4)$ & $1(0.5)$ & $1(0.5)$ & & \\
\hline Surgery & Yes & No & NA & - & - & - \\
\hline n (\%) & $130(70)$ & $27(14.4)$ & $30(15.6)$ & - & - & - \\
\hline Metastasis site & Lung & Liver & Lymph node & Bone & Peritoneum & Multiple \\
\hline n (\%) & $10(5.3)$ & $47(25.1)$ & $28(15)$ & $6(3.2)$ & 45 (24.1) & $38(20.3)$ \\
\hline BMI & $\begin{array}{l}\text { Mean } \pm \text { SD } \\
22.5 \pm 4.14\end{array}$ & $\begin{array}{l}\text { Median (min-max) } \\
22.0(17-32)\end{array}$ & - & - & - & - \\
\hline
\end{tabular}

Table.2. Clinico-pathologic characteristics of the patients according to SII groups.

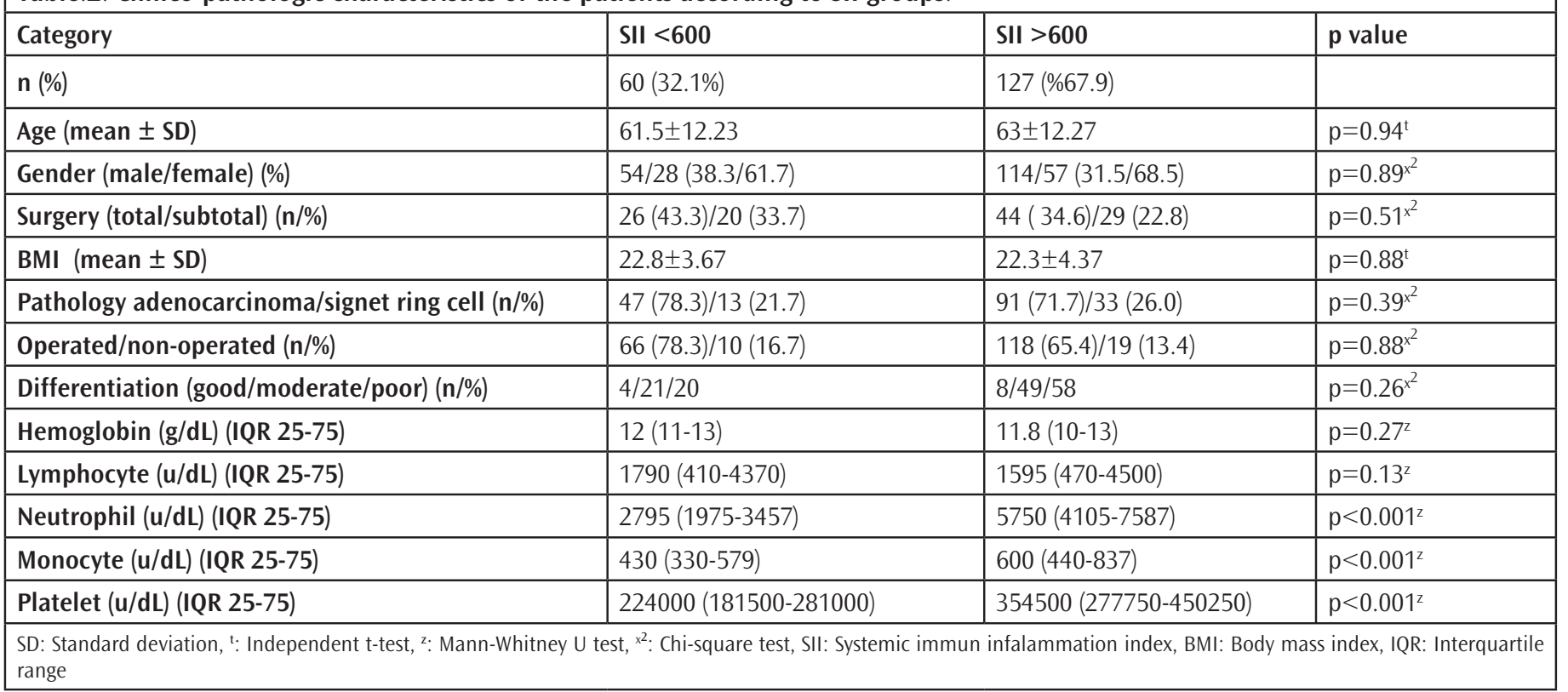


group and 9 (6.9-11.0) months in the high SII group. Although no statistically significant difference was observed in survival, it was 3 months longer in the low SII group (Figure 2).

No significant difference was found in age, gender, body mass index, histological subtype, previous gastric surgery, and pretreatment elevation in markers between the patients in the low and high SII groups (Table 2).

\section{DISCUSSION}

In our study, we found that a low SII value was associated with a better survival in patients with metastatic gastric cancer, even though it was not statistically significant.

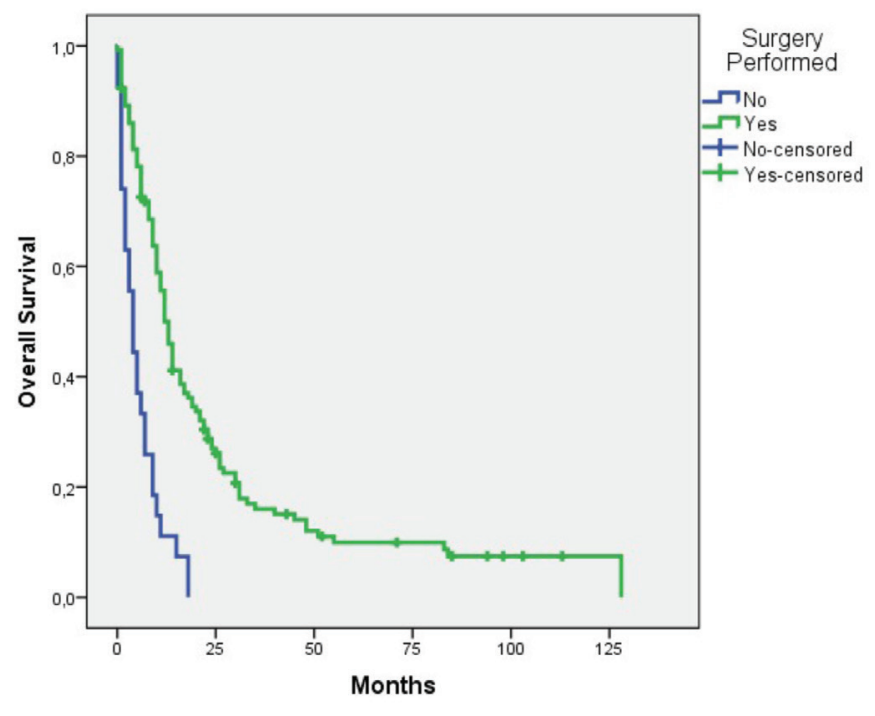

Figure 1. Kaplan-Meier survival curve according to the surgical procedure

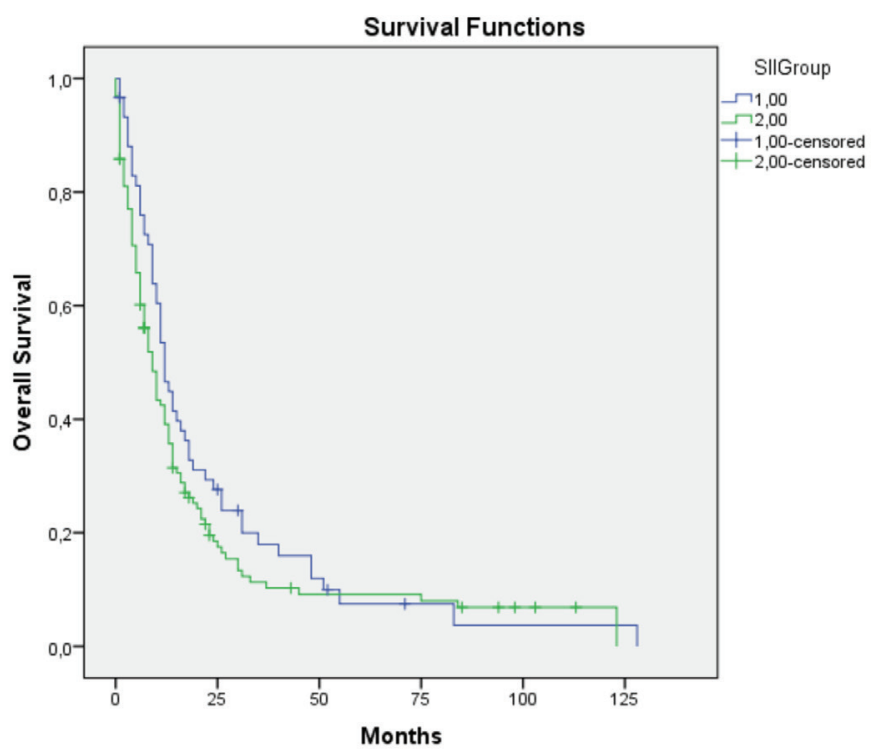

Figure 2. The effect of SII on OS in metastatic gastric cancer SII: Systemic immune-inflammation index, OS: Overall survival
Recent studies have shown that systemic immune response stimulates malignant tumor development, is associated with a more aggressive clinical course and shorter survival, and predicts prognosis in patients with malignant tumors. SII is used in practice and has been reported to show immune and inflammatory status in various tumors in the preoperative period and can predict prognosis (20-22). SII is calculated with the formula - $\mathrm{Px}(\mathrm{N} / \mathrm{L})$. It is known that $\mathrm{N}$ help tumor cells escape immune surveillance and, thus, the development of invasion, proliferation, and metastasis while inhibiting the proliferation and migration of tumor cells in L. With this mechanism, a high SII value shows a stronger inflammatory response but a weaker immunity and can be used as an applicable inflammation index in clinical practice that can predict cancer prognosis.

Previous studies showed that SII is associated with prognosis during the preoperative and neoadjuvant treatment period in patients with gastric cancer, but as far as we know, there is no study evaluating SII focused on a patient group with metastatic gastric cancer.

Table 3. Clinical characteristics and survival of the study group

\begin{tabular}{|c|c|c|c|}
\hline & $\mathbf{n}$ & $\begin{array}{l}\text { Median OS ( } 95 \% \\
\text { confidence interval) }\end{array}$ & $p$ value \\
\hline \multicolumn{4}{|l|}{ Gender } \\
\hline Female & 63 & $10(8.32-11.6)$ & \multirow{2}{*}{$p=0.97 *$} \\
\hline Male & 124 & $10(7.52-12.4)$ & \\
\hline \multicolumn{4}{|l|}{ Age (years) } \\
\hline$<65$ years & 115 & $12(8.6-15.3)$ & \multirow{2}{*}{$p=0.61^{*}$} \\
\hline$>65$ years & 72 & $9(6.5-11.4)$ & \\
\hline \multicolumn{4}{|l|}{ Pathology } \\
\hline Adenocarcinoma & 138 & $11(8.7-13.2)$ & \multirow{3}{*}{$p=0.28^{*}$} \\
\hline Signet ring cell & 46 & $9(5.43-12.5)$ & \\
\hline Mucinous & 1 & $26(8.23-11.7)$ & \\
\hline \multicolumn{4}{|l|}{ Differentiation } \\
\hline Good & 7 & $21(0.36-41.6)$ & \multirow{3}{*}{$p=0.26^{*}$} \\
\hline Moderate & 45 & $10(5.7-14.2)$ & \\
\hline Poor & 53 & $12(9.06-14.9)$ & \\
\hline \multicolumn{4}{|l|}{ SII } \\
\hline$<600$ & 60 & $12(15.3-30.6)$ & \multirow{2}{*}{$p=0.13^{*}$} \\
\hline$>600$ & 127 & $9(13.9-25.8)$ & \\
\hline \multicolumn{4}{|l|}{ Marker elevation } \\
\hline Yes & 140 & $12(11.3-22.6)$ & \multirow{2}{*}{$p=0.06^{*}$} \\
\hline No & 107 & $17(9.3-14.6)$ & \\
\hline \multicolumn{4}{|l|}{ Surgery } \\
\hline Yes & 130 & $13(11.1-14.8)$ & \multirow{2}{*}{$p<0.001^{*}$} \\
\hline No & 27 & $4(2.3-5.6)$ & \\
\hline
\end{tabular}


The study conducted by Wang et al. (20) in patients with gastric cancer found that a high SII value was associated with poor prognosis in stage 1-3 patients with cancer. It also showed that high SII values were associated with advanced age, advanced stage, poor histological subtype, increased local lymph node involvement, and distant metastasis. Again, this study revealed no significant relationship between SII and survival in stage 4 patients. Similarly, in our study, no statistically significant relationship was found between survival and SII in patients with metastatic gastric cancer. However, survival was shorter in the high SII group (20).

Chen et al. (6) evaluated the prognostic significance of SII in the pre-neoadjuvant period in patients with advanced gastric cancer, where patients with metastatic cancer were not included. Moreover, they found that patients with low SII levels had a higher survival rate and were better in terms of 1-3 and 5-year disease-free survival compared to the group with high SII. They also showed that SII is an independent prognostic factor (6).

A meta-analysis conducted by Sun et al. (23) on patients with metastatic gastric cancer who underwent palliative gastric surgery concluded that gastrectomy significantly and positively affected survival. In our study, in line with the results of this meta-analysis, the median survival was statistically significantly better in patients who underwent primary surgery than those who did not (13 months vs. 4 months; $p<0.001$ ).

Serum tumor markers are tests used in the evaluation of response to the treatment and during the follow-up in patients with gastric cancer. Various studies have reported that high carcinoembryonic antigen (CEA) levels at the time of diagnosis are an independent prognostic factor and associated with a shorter survival $(24,25)$. In our study, patients with high serum CEA levels exhibited shorter survival as compared to the group with low levels, but they did not reach the limit of significance, which was attributed to the low number of cases.

\section{Study Limitations}

There were several limitations to our study. First, it was designed as a retrospective, single-center study and had a limited number of patients. Second, although SII is an independent predictor in many tumors, its sensitivity and specificity are not high. In future, prospective, randomized, and well-designed studies are needed to optimize the appropriate cut-off value.

\section{CONCLUSION}

SII is an effective, easily applicable, reproducible, and inexpensive marker, and can be used in patients at an early and advanced stage cancer to show prognosis. In our study, we found that a high SII value was associated with poor survival, although not statistically significant. It was also found that patients with metastatic gastric cancer who underwent primary surgery exhibit higher survival. However, larger prospective studies are needed to clarify the prognostic value of SII in patients with metastatic gastric cancer.

\section{Ethics}

Ethics Committee Approval: Ethical approval, date: 23/07/2020/07, decision number: 126, was obtained from the Ethics Committee of Kayseri Training and Research Hospital.

Informed Consent: Consent was obtained from the patients that the file information would be used before the study.

Peer-review: Externally and internally peer-reviewed.

\section{Authorship Contributions}

Concept: H.D., Design: H.D., M.I., Data Collection or Processing: H.D., I.B., Y.K., Analysis or Interpretation: H.D., I.B., Literature Search: H.D., S.K.E., M.I.., Writing: H.D.

\section{REFERENCES}

1. Siegel RL, Miller KD, Jemal A. Cancer statistics, 2019. CA Cancer J Clin 2019;69:7-34

2. Bray F, Ferlay J, Soerjomataram I, Siegel RL, Torre LA, Jemal A. Global cancer statistics 2018: GLOBOCAN estimates of incidence and mortality worldwide for 36 cancers in 185 countries. CA Cancer J Clin 2018;68:394-424.

3. Torre LA, Siegel RL, Ward EM, Jemal A. Global Cancer Incidence and Mortality Rates and Trends--An Update. Cancer Epidemiol Biomarkers Prev 2016;25:16-27.

4. Ma J, Yao S, Li XS, Kang HR, Yao FF, Du N. Neoadjuvant Therapy of DOF Regimen Plus Bevacizumab Can Increase Surgical Resection Ratein Locally Advanced Gastric Cancer: A Randomized, Controlled Study. Medicine (Baltimore) 2015;94:e1489.

5. Mackenzie M, Spithoff K, Jonker D. Systemic therapy for advanced gastric cancer: a clinical practice guideline. Curr Oncol 2011;18:e202-9.

6. Chen L, Yan Y, Zhu L, Cong X, Li S, Song S, et al. Systemic immuneinflammation index as a useful prognostic indicator predicts survival in patients with advanced gastric cancer treated with neoadjuvant chemotherapy. Cancer Manag Res 2017;9:849-67.

7. Park SC, Chun HJ. Chemotherapy for advanced gastric cancer: review and update of current practices. Gut Liver 2013;7:385-93.

8. Piazuelo MB, Riechelmann RP, Wilson KT, Algood HMS. Resolution of Gastric Cancer-Promoting Inflammation: A Novel Strategy for Anticancer Therapy. Curr Top Microbiol Immunol 2019;421:319-59.

9. Bang CS, Lee JJ, Baik GH. Prediction of Chronic Atrophic Gastritis and Gastric Neoplasms by Serum Pepsinogen Assay: A Systematic Review and Meta-Analysis of Diagnostic Test Accuracy. J Clin Med 2019;8:657.

10. Elinav E, Nowarski R, Thaiss CA, Hu B, Jin C, Flavell RA. Inflammationinduced cancer: crosstalk between tumors, immune cells, and microorganisms. Nat Rev Cancer 2013;13:759-71. 
11. Kim KH, Kwon HC, Oh SY, Kim SH, Lee S, Kwon KA, et al. Clinicopathologic significance of ERCC1, thymidylate synthase and glutathione S-transferase P1 expression for advanced gastric cancer patients receiving adjuvant 5 -FU and cisplatin chemotherapy. Biomarkers 2011;16:74-82.

12. Diakos Cl, Charles KA, McMillan DC, Clarke SJ. Cancer-related inflammation and treatment effectiveness. Lancet Oncol 2014;15:e493503.

13. Borsig L, Wolf MJ, Roblek M, Lorentzen A, Heikenwalder M. Inflammatory chemokines and metastasis--tracing the accessory. Oncogene 2014;33:3217-24.

14. Mori M, Shuto K, Kosugi C, Narushima K, Hayashi H, Matsubara H, et al. An increase in the neutrophil-to-lymphocyte ratio during adjuvant chemotherapy indicates a poor prognosis in patients with stage II or III gastric cancer. BMC Cancer 2018;18:1261.

15. Hu B, Yang XR, Xu Y, Sun YF, Sun C, Guo W, et al. Systemic immuneinflammation index predicts prognosis of patients after curative resection for hepatocellular carcinoma. Clin Cancer Res 2014;20:621222.

16. Hong X, Cui B, Wang M, Yang Z, Wang L, Xu Q. Systemic Immuneinflammation Index, Based on Platelet Counts and NeutrophilLymphocyte Ratio, Is Useful for Predicting Prognosis in Small Cell Lung Cancer. Tohoku J Exp Med 2015;236:297-304.

17. Passardi A, Scarpi E, Cavanna L, Dall'Agata M, Tassinari D, Leo S, et al. Inflammatory indexes as predictors of prognosis and bevacizumab efficacy in patients with metastatic colorectal cancer. Oncotarget 2016;7:33210-9.

18. Geng Y, Shao Y, Zhu D, Zheng X, Zhou Q, Zhou W, et al. Systemic ImmuneInflammation Index Predicts Prognosis of Patients with Esophageal
Squamous Cell Carcinoma: A Propensity Score-matched Analysis. Sci Rep 2016;6:39482.

19. Shi H, Jiang Y, Cao H, Zhu H, Chen B, Ji W. Nomogram Based on Systemic Immune-Inflammation Index to Predict Overall Survival in Gastric Cancer Patients. Dis Markers 2018;2018:1787424.

20. Wang K, Diao F, Ye Z, Zhai E, Ren H, Li T, et al. Prognostic value of the systemic immune-inflammation index in patients with gastric cancer. Chin J Cancer 2017;36:75.

21. Aziz MH, Sideras K, Aziz NA, Mauff K, Haen R, Roos D, et al. The Systemicimmune-inflammation Index Independently Predicts Survival and Recurrence in Resectable Pancreatic Cancer and its Prognostic Value Depends on Bilirubin Levels: A Retrospective Multicenter Cohort Study. Ann Surg 2019;270:139-46.

22. Gao Y, Zhang H, Li Y, Wang D, Ma Y, Chen Q. Preoperative increased systemic immune-inflammation index predicts poor prognosis in patients with operable non-small cell lung cancer. Clin Chim Acta 2018;484:272-7.

23. Sun J, Song Y, Wang Z, Chen X, Gao P, Xu Y, et al. Clinical significance of palliative gastrectomy on the survival of patients with incurable advanced gastric cancer: a systematic review and meta-analysis. BMC Cancer 2013;13:577.

24. Uda H, Kanda M, Tanaka C, Kobayashi D, Inaoka K, Tanaka Y, et al. Perioperative Serum Carcinoembryonic Antigen Levels Predict Recurrence and Survival of Patients with Pathological T2-4 Gastric Cancer Treated with Curative Gastrectomy. Dig Surg 2018;35:55-63.

25. Wang K, Jiang X, Ren Y, Ma Z, Cheng X, Li F, et al. The significance of preoperative serum carcinoembryonic antigen levels in the prediction of lymph node metastasis and prognosis in locally advanced gastric cancer: a retrospective analysis. BMC Gastroenterol 2020;20:100. 